\title{
Dissonanzen im transatlantischen Bündnis überwinden
}

\author{
Friedbert Pflüger*
}

\begin{abstract}
The discord in transatlantic relations, erupted over Iraq, has brought older and more fundamental differences between Europe and the United States into focus. It also uncovered diverging developments and longstanding misunderstandings which will not be overcome quickly. In talking about a »Reconstruction of the West « and a »new Trans-Atlanticism «, even German Foreign Minister Fischer admitted how heavily this relationship is burdened but stayed short of offering remedies. This essay points out several ways to strengthen the German-American relations and the transatlantic partnership in general. Strengthening NATO, living up to the European Security Strategy, joint responsibility, building on common tasks, evolving the transatlantic economic linkage and promoting publicly the value of the partnership will help resolve the dissonances in this historic alliance.
\end{abstract}

Keywords: Transatlantische Partnerschaft, gemeinsames Handeln, Stärkung der NATO, Europäische Sicherheitsstrategie (ESS), transatlantischer Binnenmarkt, öffentliches Eintreten.

\section{Annus horribilis 2003}

) merika hatte seinen Flirt mit der Hegemonie; und Europa hatte seinen Flirt mit moralischer Selbstgerechtigkeit." Mit diesen Worten fasste am 2. Juni 2004 der einstige Außenminister der USA, Henry Kissinger, in Berlin das jüngste Zerwürfnis in den transatlantischen Beziehungen trefflich zusammen und erkannte Gründe dafür auf beiden Seiten des Atlantiks. Davon lag ein gravierender in Europa: In einem entscheidenden Augenblick für die transatlantischen Partner war Europa »nicht dialogfähig, wo wir es hätten sein müssen, als die Konflikte aufbrachen, was dann definitiv in der Irak-Krise der Fall war «, wie es Außenminister Fischer am 6. März 2004 in der FAZ eingestand. Kissingers Umschreibung ist fast schon britisches Understatement, denn in der Tat erlebten die transatlantischen Beziehungen in $2003 \mathrm{ihr}$ »annus horribilis«. Dabei spalteten die Auseinandersetzungen über das richtige Vorgehen in der Irak-Frage nicht nur die transatlantische Partnerschaft, sondern obendrein auch noch Europa in »alt « und »neu $\ll$.

Wenn es eine Lehre gibt, die alle Beteiligten diesseits und jenseits des Atlantiks aus 2003 gelernt haben sollten, dann die, dass wir gute und stabile transatlantische Beziehungen nicht als einen konstanten Wert an sich, und damit einfach als gegeben, betrachten dürfen. Auch für diese Beziehungen müssen wir arbeiten und uns einsetzen. Diese Einsicht ist nicht neu. Deutschland hat in der Vergangenheit es stets in seinem ureigensten Interesse als seine historische Aufgabe betrachtet, Europa und die USA in der transatlantischen Allianz zu halten, ein Abdriften Kontinentaleuropas zu verhindern und es nicht zum Gegengewicht oder sogar Gegenpol der USA werden zu lassen. Darin lag die Erkenntnis, dass die erfolgreichste Allianz in der Geschichte nicht nur eine Wertegemeinschaft bildete, sondern nur geschlossen die globalen Herausforderungen meistern und gestalten konnte.

\footnotetext{
* Dr. Friedbert Pflüger, MdB, Außenpolitischer Sprecher der CDU/CSUBundestagsfraktion und Mitglied im CDU-Bundesvorstand.
}

2003 hat auch gezeigt, dass die Zeit der traditionellen Beschwörungen und Rituale vorbei ist. Die Generation, die den 2. Weltkrieg noch bewusst erlebt hat und der diese Beschwörungen besonders wichtig waren, ist dabei abzutreten. Die heutigen und zukünftigen Generationen müssen noch ihre Sprache und Formen finden, mit denen sie die Beziehungen hochhalten wollen.

So sehr »der Zweck Europas etwas anderes sein muss als Widerstand gegen die USA «, wie es auch Kissinger in seiner Rede vom Juni 2004 anmahnte, so wenig ging es auch darum, dass Europa als willfähriger Adjutant der USA handelt. Vielmehr war es Ziel deutscher Regierungen von Adenauer über Brandt bis Kohl gewesen, die Balance zwischen europäischer Orientierung und atlantischer Verbindung zu wahren. Die deutsche Linke der Generation der Vietnam-Proteste hat bewusst mit dieser tradierten Politik gebrochen. Die Bundesregierung lehnte öffentlich und rhetorisch überhöht jegliche Irak-Intervention zur Durchsetzung von VN-Resolutionen unilateral ab und sammelte fortan weltweit Stimmen gegen die eigenen Bündnispartner. Unbestritten gab es sehr gute Gründe gegen ein militärisches Vorgehen und es war legitim, von einem Krieg abzuraten. Dies hätte jedoch geschehen können, ohne Ressentiments zu schüren. Jedoch sahen die Anhänger von Rot-Grün in Schröders »deutschem Weg « die notwendige »Unabhängigkeitserklärung « von den USA, was ein seltsames Verständnis der transatlantischen Beziehungen offenbart, und sahen geflissentlich über die Doppelmoral der Regierungsparteien hinweg, deren Vertreter einerseits den Irak-Krieg als völkerrechtswidrig brandmarkten, während andererseits die von ihnen getragene Bundesregierung Beihilfe zum Krieg leistete, indem sie Überflugrechte gewährte und US-Kasernen bewachen ließ. Diese Entwicklung fasste Harvard-Professor Joe Nye am 23. April 2003 in der FAZ, in dem er auf die beiden traditionellen Standbeine europäische Orientierung und atlantische Verbindung anspielte, mit den Worten zusammen: »Die Regierung Schröder hat im Vergangenen offenkundig entschieden, dass eine Bein (die atlantische Verbindung) weg zu hauen. « 


\section{Ursachen der politischen Dissonanzen}

Um das Verhältnis zwischen Europa und den Vereinigten Staaten von Amerika nach den Ereignissen der vergangenen Jahre wieder auf eine starke Grundlage zu stellen, dürfen und können die Beteiligten nicht einfach zur Tagesordnung übergehen. Das »annus horribilis « hatte seine Ursachen. Diese liegen in langfristigen Entwicklungen, die in der europäischen Revolution von 1989/1990 ihren Ausgang nahm, und in augenblicklichen Befindlichkeiten diesseits und jenseits des Atlantiks. Mit beiden müssen wir uns auseinandersetzen.

\subsection{Globale Trends}

$\mathrm{Zu}$ Beginn des 21. Jahrhunderts klafft in weiten Teilen das Bedrohungsgefühl in Europa und in Amerika auseinander. Während des Kalten Krieges gab es auf beiden Seiten zwangsläufig eine sehr ähnliche Gefahrenanalyse. Nicht zuletzt ging es um Europa selbst. Mit Wegfall des Eisernern Vorhangs löste sich die Kohärenz des strategischen Denkens zwischen beiden Seiten des Atlantiks auf. Die Welt wurde zugleich in den 90er Jahren immer komplizierter und chaotischer. Schon Januar 1993 sprach der britische Außenminister Douglas Hurd von »the new disorder«. Zahlreiche ethnische, nationalistische und religiöse Konflikte, die die Blockkonfrontation unterdrückt hatte, brachen hervor. Die Kriege auf dem Balkan waren dafür symptomatisch. Die neue Unordnung war Ausdruck gravierender Veränderungen des Sicherheitsumfeldes, die schlagartig in den grauenhaften Anschlägen des 11. Septembers 2001 sichtbar wurden.

Erstens haben Globalisierung und die Auflösung der Bipolarität eine Multiplizierung der Akteure in den internationalen Beziehungen gefördert. Im 21. Jahrhundert sind plötzlich nicht-staatliche Organisationen oder sogar Einzelpersonen fähig und willens, die nationale bzw. internationale Sicherheit ernsthaft $\mathrm{zu}$ bedrohen. Al-Qaida als bestes Beispiel für den neuartigen transnationalen Terrorismus ist darauf aus, Massenmorde zu begehen. Zweitens hat die räumliche Entfernung zu einer Krise im Prinzip an Bedeutung verloren. So wird heute die Sicherheit Europas nicht mehr aus der europäischen Sicherheitszone heraus in Frage gestellt, sondern von außerhalb. Folglich können wir unsere Sicherheit nicht mehr mit klassischer Grenzverteidigung allein gewährleisten. Nährboden für die heutigen Bedrohungen liefern Zonen der Instabilität und Ordnungslosigkeit, für die das Afghanistan der Taliban das beste Beispiel ist. Terroristen nutzen diese Zonen skrupellos als Operationsbasis, Rekrutierungspool und Finanzierungsquelle für die Verfolgung ihrer menschenverachtenden Ziele aus. Drittens haben die Anschläge vom 11. September die Verwundbarkeit demokratischer Gesellschaften aufgezeigt. Heute steht die asymmetrische Kriegsführung mit ihren Angriffen auf weiche Ziele, wie z.B. die Ölversorgung, die Finanzzentren oder öffentliche Versorgungseinrichtungen, im Mittelpunkt der sicherheitspolitischen Aufmerksamkeit.

\subsection{Reaktionen in den USA und Europa}

Weite Teile Europas haben sich dieser Entwicklung nicht bewusst gestellt. Vielmehr forderten viele Europäer nach der Aufhebung des Eisernen Vorhangs eine Friedensdividende. Damit ging das Gefühl einher, den amerikanischen Schutzschild nicht mehr zu benötigen. Die USA hingegen haben relativ schnell, das heißt schon unter den Präsidenten Bush sen. und Clinton, über die Anpassung der amerikanischen Sicherheitsstrategie an die neuen Herausforderungen nachgedacht. Dies blieb in Europa weitestgehend unbeachtet. Die amerikanische Diskussion drehte sich dabei darum, unter Beibehaltung der traditionellen Ziele, Unverwundbarkeit und hegemoniale Stellung zu wahren, Gefahren aktiv und nicht erst reaktiv anzugehen sowie einen status quo, der nicht hinnehmbar ist, zu überwinden. In Europa hingegen herrschte und herrscht eine größere Bereitschaft, den status quo von Machtkonstellationen zu akzeptieren.

Der 11. September hat die grundlegende Neuausrichtung der USA lediglich beschleunigt. Das Ereignis hat eine enorme psychologische Wirkung in den USA verursacht. Einen Angriff auf das eigene kontinentale Territorium hatten die USA seit dem amerikanisch-britischen Krieg von 1812-1814 nicht gekannt. Nun wurde dem mächtigsten Land der Welt seine Verwundbarkeit auf einmal drastisch vor Augen geführt und seit dem tausende Male im Fernsehen wiederholt. Dass die Neuausrichtung kein Steckenpferd der republikanischen Administration war, belegte eine der führenden außenpolitischen Stimmen der Demokraten in den USA, Richard Holbrooke, der schon frühzeitig darauf hinwies, dass bei aller berechtigten Kritik an Präsident Bush auch eine von den Demokraten gestellte Regierung im wesentlichen die gleiche Politik verfolgt hätte. So kommt nicht überraschend, dass der demokratische Präsidentschaftskandidat John Kerry den Krieg gegen Terrorismus und die Irak-Politik sogar noch kraftvoller fortsetzen will. Im Januar 2004 sagte er in einer Grundsatzrede, dass er »die Sicherheit des Landes niemals irgendeiner Institution überantworten « und die nationale Sicherheitsstrategie nicht »von jenen bestimmen lassen werde, die sich reflexartig gegen jede amerikanische Militärintervention irgendwo auf der Welt wenden«. Im Mai 2004 ergänzte er, dass er notfalls militärische Gewalt auch präventiv zum Schutz amerikanischer Interessen einsetzen werde. Diese Aussagen bekräftigte Kerry in seiner Rede auf dem Parteitag der Demokraten Ende Juli 2004 in Boston, in der er Amerika das Recht auf unilaterale Gewalt vorbehielt. Er werde »nie zögern, Gewalt einzusetzen, wenn es erforderlich ist «. Auch wenn es in den USA schon bald nach Ende des Irak-Krieges wachsende Kritik an Präsident Bush gab, das grundlegende Bedrohungsgefühl gegenüber islamistischem Terrorismus und Massenvernichtungswaffen sowie die Entschlossenheit, gegen die Bedrohung der USA vorzugehen, sind in beiden großen politischen Lagern der USA ausgeprägt. Deshalb stünde ein gewählter Präsident Kerry in der Sicherheitspolitik für Kontinuität.

Die Auswirkungen des 11. September in Europa waren nicht vergleichbar. Nach der anfänglichen Betroffenheit, dem Mitgefühl und der Beschwörung von »uneingeschränkter 
Solidarität « (so Bundeskanzler Gerhard Schröder) ging man bald zur Tagesordnung über. »9/11 « hat zumindest in Deutschland im öffentlichen Bewusstsein zu keinem nachhaltigen Gefühl wachsender Gefahren geführt. Wem ist noch bewusst, dass nach wie vor der NATO-Bündnisfall gilt, der im Herbst 2001 kurz nach den Anschlägen ausgerufen wurde? Vielmehr geht eine weit verbreitete Einschätzung davon aus, dass dies ein Angriff von Terroristen auf Amerika war, und dass Europa wohl kaum ein Zielpunkt terroristischer Anschläge sein würde - es sei denn man solidarisiere sich zu stark mit Washington. Selbst die furchtbaren Anschläge von Madrid am 11. März 2004 haben die Bevölkerung Europas nicht aufgerüttelt und ein Umdenken eingeleitet. Die Fehleinschätzung, man könne sich auf eine »Insel der Glückseligen « zurückziehen und sich durch Beschwichtigung und Distanzierung von seinen Partnern Sicherheit erkaufen, ist indes eine schreckliche Illusion. Beschwichtigung als Konzept hat jedoch noch nie in der Geschichte im Angesicht einer totalitären Bedrohung funktioniert. Mit Madrid haben Al-Qaida, seine Ableger und Nachahmer demonstriert, dass sie gedenken, mit Ängsten und Wahrnehmungen zu spielen. Sie wollen damit ganz bewusst einen Keil in das westliche Bündnis treiben.

Bundesminister Fischer hat seit September 2003 mehrfach öffentlich zugegeben - seine zitierte Äußerung vom 06.03.2004 geht in diese Richtung -, dass es ein entscheidender Fehler gewesen sei, dass Amerika und Europa nicht sofort nach dem 11. September eine umfassende strategische Diskussion geführt haben. Während sich Europa die Bedrohungslage schön geredet hat, wurde in den USA eine neue Nationale Sicherheitsstrategie (NSS) entwickelt und im September 2002 veröffentlicht. Hätte es darüber sofort eine ernsthafte Debatte gegeben, dann wären viele Missverständnisse und Konflikte zwischen den transatlantischen Partnern vermieden worden. Anstatt eigene Alternativen auf die neuen Fragestellungen zu entwickeln, übte sich Europa in der Dämonisierung amerikanischer Antworten. Erst am 12./13. Dezember 2003 auf dem Europäischen Rat in Brüssel verabschiedete die EU eine gemeinsame Sicherheitsstrategie (ESS) und damit eine tragfähige Antwort auf die NSS. Nach all den rhetorischen Schlagabtauschen verblüfft umso mehr die große Gemeinsamkeit zwischen beiden Ansätzen. Ein Beispiel: Wenn auch mit anderen Worten spricht sich auch die ESS im Ernstfall ebenfalls für ein präemptives militärisches Eingreifen als äußerstes Mittel aus.

\subsection{Gesellschaftliche Differenzen und Gemein- samkeiten der Partner}

Zur gleichen Zeit, in denen die strategischen Wahrnehmungen auseinander gingen, haben aber auch zwei gesellschaftliche Phänomene verstärkt zu Unstimmigkeiten im transatlantischen Verhältnis geführt.

Trotz aller Nähe und Affinität lässt das landläufige fundierte Wissen diesseits und jenseits des Atlantiks über die jeweilig andere Seite zu wünschen übrig. Über so manche Lücke im Wissen der Amerikaner über Europa wird oft gelästert. Aber die von Europäern gepflegten Vorurteile über Amerika -
Land des Fast Food, der hemmungslosen Kapitalisten, der Kulturlosigkeit, der Schießwütigen - sind letztlich ebenso Ausdruck von Unkenntnis. Gleichzeitig sind viele vom American way of life angezogen. Gerne halten sich Europäer für Amerika-Experten und nehmen vorwiegend doch nur das vermeintliche Amerika wahr, das ihnen zusagt. Deshalb erfährt der US-Autor und Filmemacher Michael Moore so viel Beachtung in Europa und wird Präsident Bush derart abgelehnt. Wer erinnert sich, dass der in Deutschland so beliebte ehemalige Präsident Clinton im Dezember 1998 den Irak bombardieren ließ oder im August 1998 Raketenangriffe gegen Khartum und Afghanistan befahl? Diese Diskrepanz erschwert politisch Handelnden, transatlantische Positionen zu vermitteln umso mehr, wenn andere aus wahltaktischen Gründen mit den diffusen Gefühlen spielen.

Amerika ist eine von ungebrochener Dynamik und von $\mathrm{Zu}$ kunftsglauben geprägte Nation, die nicht nur politisch, militärisch und wirtschaftlich, sondern inzwischen eben auch kulturell - und ich meine nicht Massenkultur - weltweit führend ist. Ohne die großen innerstaatlichen Differenzen vergessen zu wollen, ist Amerika zugleich im Schnitt mit Abstand traditionellen Werten stärker verhaftet als alle anderen westlichen Staaten, mit Ausnahme Irlands. In punkto Patriotismus und Religiosität steht Amerika der Türkei näher als Deutschland oder selbst dem Vereinigten Königreich. Das amerikanische Sendungsbewusstsein, das in Europa besonders auf Unverständnis stößt, und die überragende Bedeutung von Freiheit sind untrennbar mit der (Staats-) Gründung und dem Selbstverständnis der USA verknüpft. Die ersten Siedler waren schließlich in Europa religiös und politisch Verfolgte gewesen, die Amerika als Gegenbild zu Europa definierten. Laut einer letzten Studie der Universität von Michigan über Werte in der Welt ist Amerika Ende des 20. Jahrhunderts noch traditioneller geworden. In Amerika verläuft die politische Anbindung weniger entlang des Einkommens als vielmehr entlang von Werten, weshalb auch die Wahlkämpfe in Amerika primär entlang der Werteeinstellung geführt werden. Es lassen sich beliebig viele weitere Beispiele nennen, bis hin zu unterschiedlichen Begrifflichkeiten wie zum Beispiel bei »war «/Krieg. In den USA wird der Begriff auch im abstrakten Sinne von Kampf verwendet. Für Europäer und speziell für Deutsche beschwört der Terminus Krieg hingegen negative kollektive Erinnerungen und Ängste.

Als zweiter Trend ist zu beobachten, dass Amerika und Europa sich hinsichtlich ihrer Innovationsfähigkeit und ökonomischen Kraft sehr unterschiedlich entwickeln. In der EU leben heute hundert Millionen Menschen mehr als in der Vereinigten Staaten. Wenn die gegenwärtigen Trends anhielten, würden die USA in den nächsten vier Jahrzehnten mehr Einwohner als die selbst erweiterte EU zählen. Während heute das mittlere Alter der Menschen in den USA und Europa bei 36 bzw. 38 Jahren liegt, würde das Durchschnittsalter im Jahre 2015 in Amerika bei 36,2, in Europa bei 52,7 Jahren liegen. Der Prozess der stetigen Alterung der Gesellschaft hätte nachhaltig Auswirkungen auf die Dynamik der europäischen Volkswirtschaften. Gleichzeitig ist der Wille zu unternehmerischer Selbständigkeit in Amerika 
weitaus stärker. In Europa ziehen es 50 Prozent der Menschen vor, als Angestellter und nicht als Unternehmer zu arbeiten. In den USA sagen dies nur 29 Prozent. Eine Unternehmensgründung in den USA brauche im Schnitt 4 Tage, in Deutschland dauert sie 45 Tage. Auch die Arbeitskultur ist sehr unterschiedlich. Im Jahre 1982 arbeiteten Amerikaner und Europäer in etwa die gleiche Stundenzahl im Jahr. Heute arbeitet man in Amerika 300 Stunden pro Kopf pro Jahr mehr. Da auch die Produktivitätsfortschritte in Amerika größer sind als in Europa, bedeutet diese Zahl fast zwangsläufig, dass europäische Unternehmen weniger konkurrenzfähig werden. Dank dieser Innovationsfreudigkeit gelang es den Amerikanern auch viel früher, sich dem Globalisierungsschub anzupassen - mit der Folge, dass die Globalisierung zu Unrecht als ein amerikanisches Phänomen wahrgenommen wird.

Mit diesen gesellschaftlichen Trends soll nicht einer zunehmenden Entfremdung der transatlantischen Partner das Wort geredet werden. Nach wie vor gilt: Die transatlantische Gemeinschaft gründet auf einem weltweit einzigartigen Fundament gemeinsamer Werte, vergleichbarer Zivilgesellschaften sowie dem gemeinsamen Streben nach internationaler Durchsetzung von Demokratie, Menschenrechten, individuellen Freiheiten und Marktwirtschaft. Keine anderen zwei Regionen auf dieser Welt sind derart miteinander verknüpft und wirtschaftlich verflochten. Vielmehr geht es darum, das Verständnis für zwei Einsichten zu schärfen: Zum einen hat es zwischen den Vereinigten Staaten und Europa immer Differenzen in Werten und in Methoden, um gemeinsame Ziele zu erreichen, gegeben, zum anderen benötigen die transatlantischen Beziehungen die genaue Kenntnis vom und das Verstehen des jeweilig Anderen.

\section{Wege zur Bekräftigung}

Inzwischen denken Heerscharen darüber nach, wie die transatlantischen Beziehungen gerettet werden können. Schlagwörter machen die Runde. Außenminister Fischer sprach am 6. März 2004 in der FAZ von der notwendigen »Rekonstruktion des Westens « und »neuen Transatlantismus «. Was das heißt, sagte er nicht. Politik ist jedoch konkret. Dabei sind fünf Aspekte wesentlich.

1. Die transatlantische Gemeinschaft kann ihrem Anspruch nur dann gerecht werden, wenn sie sich den sich wandelnden Herausforderungen stellt. Dabei muss vor allem Europa die neuen Realitäten wahrnehmen und seine Mitverantwortung für die Lösung der außereuropäischen Sicherheitsprobleme stärker annehmen. Es genügt nicht, sich wortgewaltig aufzublasen, den USA Unilateralismus, Imperialismus, Bruch des Völkerrechts und Kriegslüsternheit vorzuwerfen, und gleichzeitig insgeheim darauf zu vertrauen, dass die USA letztlich die Verantwortung für die Lösung von Krisen, wie aktuell im Sudan, übernehmen. Es geht auch nicht, multilaterales Handeln der USA einzufordern und eigene substantielle Beiträge dazu zu verweigern. Europa muss vielmehr bereit sein, mehr in die eigene Stärke zu investieren, so dass wir durch das Gewicht unserer Beiträge bei Krisenbewältigungen partnerschaftsfähig sind und damit Mitsprache erhalten. An dieser Stelle sei mit aller Deutlichkeit wiederholt: Es geht bei der europäischen Stärke aus unserer Sicht nicht darum, Europa zum Gegenpol der USA aufzubauen, sondern vielmehr darum, die transatlantische Allianz zu stärken.

2. Unverzichtbares Element für die Stärkung der transatlantischen Beziehungen muss die Wiederbelebung der NATO sein. Die NATO muss primäres Instrument unserer globalen Sicherheitspolitik bleiben. Es muss wieder selbstverständlich werden, dass alle Mitglieder ihre eigenen sicherheitspolitischen Interessen weiterhin mit dem Wohl und Wehe der NATO identifizieren. Davon sind wir nach dem Vertrauensbruch vom Februar 2003, als Belgien, Frankreich und die Bundesregierung die von der Türkei erbetene Planung zur Unterstützung des Landes im Falle irakischer Angriffe verzögerten, immer noch weit entfernt. Das verdeutlichen die schwierigen Verhandlungen um den NATO-ISAF-Einsatz in Afghanistan bzw. die Irak-Ausbildungsmission. Aktionen, die gegen das Bündnis gerichtet sind, müssen in Zukunft unterbleiben. Dies gilt für beide Seiten. Auch die USA müssen bereit sein, sich weiterhin auf die transatlantische, multilaterale Kooperation einzulassen, und der Versuchung widerstehen, Europa zu spalten, was ihrer eigenen langjährigen Politik fundamental widersprechen würde. Letztlich haben die USA ein Interesse sowohl an einer Einheit Europas als auch an einer funktionierenden NATO. Die Unilateralisten in Washington, die der Illusion nachhängen, Amerika könne ohne dauerhafte Bündnisse seine Werte und Interessen in der Welt durchsetzen, überschätzen bei aller militärischen Stärke die Fähigkeiten der USA. Ein auf sich allein gestelltes Amerika, ab und zu durch eine »Koalition der Willigen « aus dem Werkzeugkasten der NATO verstärkt, kann zwar Kriege für sich entscheiden, aber nicht dauerhaft Frieden schaffen. Leider hat dies der Irak-Konflikt bewiesen, in dessen Nachkriegsphase sich vor allem die US-Administration taub stellte. Innerhalb der NATO mag ein unilaterales Vorgehen kurzfristig weniger Reibungen erzeugen, langfristig jedoch unterminiert es die transatlantische Stabilität.

Die Wiederbelebung der NATO muss in zweierlei Hinsicht erfolgen. Zum einen muss die NATO wieder der Ort der transatlantischen strategischen Sicherheitsdebatte werden. Mit der Europäischen Sicherheitsstrategie (ESS) ist Europa dialogfähig geworden. Jetzt gilt es eben diesen Dialog mit den USA auch mit dem Ziel zu führen, wie schon zuvor sich auf eine gemeinsame transatlantische Sicherheitsdoktrin zu einigen. Zum anderen muss Europa zusätzliche Anstrengungen unternehmen, seine militärischen Fähigkeiten signifikant zu steigern. Wer A multilaterale Einsätze fordert, muss auch B Einsatzfähigkeit und -bereitschaft sagen. Zwar gibt es bereits ermutigende Schritte im Rahmen der Europäischen Sicherheits- und Verteidigungspolitik, wie zum Beispiel der französisch-britische Vorschlag, »battle groups « einzurichten. Weitere Schritte, wie z.B. die weitere Koordinie- 
rung der Verteidigungsanstrengungen, das Ausschöpfen möglicher Synergien, das Pooling von militärischen Einheiten unter den Mitgliedsstaaten und letztlich zusätzliche finanzielle Mittel für die Streitkräfte, müssen folgen.

3. Beide Seiten des Atlantiks müssen wieder dazu übergehen, für die dringendsten geopolitischen Aufgaben gemeinsame Ziele zu formulieren und Schritte dahin abzusprechen. Gemeinsames Handeln wird entscheidend das transatlantische »Wir-Gefühl« beleben.

Afghanistan und Irak sind dafür geradezu antipodische Beispiele. Sie zeigen einmal mehr, dass Nordamerika und Europa - wenn überhaupt - nur gemeinsam in der Lage sind, gravierende Probleme zu lösen. Dazu ist notwendig, das Gefühl eines gemeinsamen Zweckes zu haben. Dies gelingt wiederum nur, wenn wir uns alle als Partner und Verbündete verstehen und faire Beiträge zur Lösung leisten, und dies gelingt nicht, wenn wir dem multilateralen Handeln eigene Beiträge verweigern.

Seit dem Frühjahr 2004 scheinen die transatlantischen Partner allmählich den Willen zum gemeinsamen Handeln wieder zu finden. So haben sich die Vorstellungen der Partner endlich angenähert, wie der Übergangsprozess im Irak zu gestalten ist. Des Weiteren haben die verschiedenen Gipfel im Juni dieses Jahres, G 8, EU, USA und NATO, gezeigt, dass die Partner ihre gemeinsame Gestaltungsaufgabe in der geopolitischen Großregion des Nahen und Mittleren Ostens und Nordafrikas begriffen haben und annehmen wollen, wenn auch im Detail manches zu wünschen übrig blieb. Meine Fraktion hat Anfang Mai einen Antrag im Bundestag für eine Partnerschaft für Frieden und Stabilität mit dieser Region eingebracht, um ihrerseits einen Beitrag zur fortlaufenden Diskussion zu leisten. In dem Antrag heben wir hervor, dass die Partnerschaft gerecht, unter Gleichen und basierend auf gegenseitigem Respekt und Verständnis gestaltet werden muss. Darunter fällt auch, dass wir in der Region wieder Glaubwürdigkeit erlangen. Dazu gehören vor allem ernsthafte Bemühungen um Fortschritte im Nahost-Friedensprozess. Jedoch darf die engere $\mathrm{Zu}$ sammenarbeit mit der Region weder Ersatz für die Friedensbemühungen sein, noch darf ein Nahost-Frieden zur Vorbedingung für die Partnerschaft gemacht werden. Entscheidend für die Glaubwürdigkeit ist aber auch die weitere Irak-Politik des Westens. Die Vorkommnisse in amerikanischen Gefangenenlagern im Irak haben nicht nur die amerikanische Glaubwürdigkeit nachhaltig erschüttert, sondern auch die des ganzen Westens. Es muss unmissverständlich klar werden, dass die transatlantischen Partner Anwendung von Folter in jeglicher Form als Verletzung der unveräußerlichen Menschenrechte verurteilen und diejenigen, die Folter begangen haben, zur Rechenschaft gezogen werden. In diesem $\mathrm{Zu}-$ sammenhang ist für europäische Schadenfreude kein Platz. Ein Erfolg in der Gestaltung des neuen Iraks ist in unser aller Interesse. Das Unternehmen Irak darf zu unserer eigenen Sicherheit nicht scheitern. Nicht zuletzt: Ein Freund in Schwierigkeiten ist ein Freund, dem man hilft.
Über die transatlantischen Di- und Konvergenzen sollte jedoch nicht vergessen werden, dass die Partner in zahlreichen Bereichen, zum Teil seit langem, lautlos und effizient zusammen arbeiten: Afghanistan - trotz aller Reibereien -, wo die NATO zum ersten Mal außerhalb Europas eingesetzt ist; der Kampf gegen den transnationalen Terrorismus und der Heimatschutz, in deren Rahmen einige Anstöße, zum Beispiel für die Stärkung der Sicherheit freien Reisens oder zur Unterbindung der Proliferation von Massenvernichtungswaffen gegeben worden sind.

Die immer wieder aufflackernde Frage des richtigen Umgangs mit dem Iran, bei der die transatlantischen Partner zuletzt Hand in Hand gingen, kann zum nächsten gravierenden Testfall für die Beziehungen werden.

Andere Themen harren noch eines transatlantischen Verständnisses, zum Beispiel die behutsame Weiterentwicklung des Völkerrechts. Die USA und die Europäer sind sich nicht mehr über die Rolle des Völkerrechts, des Multilateralismus, der Bedeutung internationaler Institutionen der richtigen Balance zwischen Diplomatie und Machtanwendung einig. Robert Kagan schreibt dies unterschiedlichen außenpolitischen Traditionen zu. Während Amerikaner die Chancen internationaler Vereinbarungen und des Völkerrechts für eine friedliche Welt besser verstehen sollten, müssen wir Europäer die Möglichkeiten und Grenzen multilateralen Vorgehens etwas nüchterner einschätzen. So ist es kein Vorzug des Völkerrechts, dass sich Staaten hinter der Staatsouveränität verschanzen können - Sudan ist das jüngste Beispiel - oder Staaten, in denen schwere Menschenrechtsverletzungen begangen werden, in den VN-Sicherheitsrat oder die VNMenschenrechtskommission hinein gewählt werden. Gefragt ist ein Multilateralismus, der handlungsfähig und effektiv ist. Diesen gilt es zu definieren.

4. Die transatlantischen Wirtschaftsbeziehungen untermauern wie kein anderer Bereich den Grad der Verbundenheit mit Zahlen: So wickelten im Mai 2004 die beiden Regionen ein Drittel des globalen Handels ab. Wir sollten die fortschreitende Verflechtung fördern und dabei langfristig auf einen einheitlichen transatlantischen Wirtschaftsraum bzw. Binnenmarkt hinarbeiten. In einem ersten Schritt müssen wir dabei lernen, besser mit unseren Streitfällen umzugehen. Die WTO und ihre Streitschlichtungsverfahren müssen verbessert und gestärkt werden. Zum Beispiel ist es ein Gedanke, die verschiedenen Kartellrechtsverfahren - Microsoft, Honeywell/GE -, die in den letzen Jahren große Aufmerksamkeit erfuhren und zu Unstimmigkeiten geführt hatten, besser zeitlich abzustimmen.

5. Nicht zuletzt verlangt die Verbesserung der transatlantischen Beziehungen, dass Politik, Wirtschaft und Kultur in Europa für diese Beziehungen werben und sich einsetzen. Es herrscht in den europäischen Öffentlichkeiten ein verzerrtes Amerika-Bild, anti-amerikanische Gefühle sind auf dem Vormarsch. Dazu haben sowohl die amerikanische Administration als auch im gehörigen Maße europäische Politiker und Medien beigetragen. Es liegt in unser aller Verantwortung, dass sich dieses Bild und dieser 
Trend nicht festsetzen. Die transatlantische Partnerschaft ist nicht etwas, das auf dem Niveau eines Allerweltsarbeitsverhältnisses dümpeln kann. Leidenschaftliches Bestreben muss es sein, den jungen Generationen ein realistisches, ausgewogenes und optimistisches Bild von Amerika zu zeigen. Dazu gehören sicherlich auch die Schattenseiten Amerikas, aber eben nicht nur. Gleiches gilt umgekehrt auch für die USA. So hat Willy Brandt in seiner Rede vor der Harvard-Universität am 5. Juni 1972 gesagt: »Sie, die Jüngeren dürfen nicht vergessen, dass die Interdependenz, die John F. Kennedy für die Staaten diesseits und jenseits des Atlantiks proklamiert hat, eine moralische, eine kulturelle, eine wirtschaftliche und politische Realität bleiben muss. "Diese Aufgabe ist in den letzten Jahren auch deshalb um so wichtiger geworden, als immer mehr in Deutschland stationierte Amerikaner abgezogen sind und noch abziehen werden, die hervorragende Botschafter und Mittler für beide Länder waren.

\section{Fazit}

Zur Jahrtausendwende schrieb das britische Magazin The Economist: »The chief question at the 1990s' end is whether Europe and America will remain a foreign-policy partnership or ignore the 20th century's lesson book and go their separate ways. "Einmal mehr bewies es seine Weitsicht. Die genannten fünf Punkte halten sich an die Lehre des 20. Jahrhunderts. Sie mögen sehr selbstverständlich klingen teilweise finden sie sich in einer kaum beachteten EUErklärung zu den transatlantischen Beziehungen vom 13. Dezember 2003 wieder - und dennoch wurden und werden sie nur unzureichend beherzigt. Dies muss sich ändern. Es ist in unserem fundamentalen Interesse, dass die transatlantische Partnerschaft fortgesetzt und in diesem Sinne die Frage des Economist beantwortet wird.

\title{
Die Krise der westlichen Allianz und die Krise des Multilateralismus
}

\author{
Joachim Krause*
}

\begin{abstract}
Since the Iraqi crisis from 2002/2003 the Atlantic Alliance has been put into question. Various authors argue that the Western Alliance has lost its substance and is becoming an empty shell. Their main argument is that the current crisis has been caused by US unilateralism, which was said to be the direct consequence of conservative and neo-conservative Republican policy strategies. This article is arguing that the causes for the transatlantic problems are considerably deeper and are preceding the Bush-Administration. These problems surely have been aggravated by the diplomacy of the BushAdministration, but they won't go away if the next election would bring about a Democratic Administration. Much of the criticism within the scholarly debate is proceeding from a simplified theoretical approach that tends to equate multilateralism with international order. It is argued in this article that one rather has to look at different expectations regarding international order on both sides of the Atlantic. While in the US multilateralism usually has been judged from its merit in terms of problem solving, Europeans have tended to value rather the procedural aspects of multilateralism (i.e. the selfbinding effect). The most important thing is to overcome this crisis by looking for new and reformed ways of multilateralism that hold the promise of both solving international problems and binding the U.S. Reforming NATO in a way that it might take over a major responsibility for international security might be a good starting point for such a political strategy.
\end{abstract}

Keywords: Transatlantische Partnerschaft, Gefährdung, Multilateralismus, Prämissen wissenschaftlicher Analysen, Perspektiven, NATO

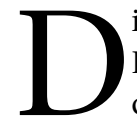
ie Irak-Krise von 2002/2003 stellt die wohl schwerste Belastung der Atlantischen Allianz seit dem Ende des Ost-West-Konflikts dar. Viele haben seither die Frage aufgeworfen, ob die Allianz überhaupt noch eine $\mathrm{Zu}$ kunft habe. Während in der Politik mittlerweile eine Sprachregelung vorherrscht, die auf größere Harmonie und Gemeinsamkeiten hindeutet, bleibt die Einschätzung der Zukunft der transatlantischen Beziehungen in der wissen-

\footnotetext{
* Prof. Dr. Joachim Krause, Direktor des Instituts für Sicherheitspolitik an der Christian-Albrechts-Universität in Kiel.
}

schaftlichen Analyse eher skeptisch. ${ }^{1}$ Viele gehen davon aus, dass die Substanz an Gemeinsamkeiten zwischen den

\footnotetext{
1 Der EU Gipfel vom 13. Dezember 2003 etwa betonte, dass die transatlantischen Beziehungen unersetzlich seien und dass sich die EU zu einer konstruktiven, ausgewogenen und zukunftsgerichteten Partnerschaft mit den USA und Kanada bekenne. Deutsche wie amerikanische Politiker betonen immer wieder die Absicht und die Notwendigkeit zusammenzuarbeiten. Dies findet sich in Dutzenden von multilateralen und bilateralen Kommuniques; vgl. Johannes Varwick, Die Zukunft der transatlantischen Sicherheitsbeziehungen, in: Österreichische Militärische Zeitschrift, 42. Jg., Heft 2, 2004, S. 141-148 ( S. 141).
} 